\title{
PECULIARITIES OF A GROUP RESPONSE OF CARDIOVASCULAR SYSTEM OF VOLUNTEERS AT DIFFERENT LATITUDES TO CHANGES OF SPACE WEATHER PARAMETERS
}

\author{
Parshina S.S. ${ }^{1}$, Samsonov S.N. ${ }^{2}$, Manykina V.I. ${ }^{2}$, Afanasyeva T.N. ${ }^{1}$, Vishnevsky V.V. ${ }^{3}$, Petrova P.G. ${ }^{4}$, \\ Petrova V.D. ${ }^{1}$, Strekalovskaya A.A. ${ }^{4}$, Tokayeva L.K. ${ }^{1}$, Kaplanova T.I. ${ }^{1}$, Potapova M.V. ${ }^{1}$ \\ ${ }^{1}$ V. I. Razumovsky Saratov State Medical University of the Russian Ministry of Health, \\ Russia, Saratov, parshinasvetlana@rambler.ru \\ ${ }^{2}$ Yu.G.Shafer Institute of Cosmophysical Research and Aeronomy of Siberian Branch of the \\ Russian Academy of Sciences, Russia, Yakutsk, s_samsonov@ikfia.ysn.ru \\ ${ }^{3}$ Institute of Mathematical Machines and Systems Problems, NSA of Ukraine, \\ Ukraine, Kiev \\ ${ }^{4}$ M.K.Ammosov Medical Institute of North-Eastern Federal University, \\ Russia, Yakutsk
}

\begin{abstract}
A simultaneous monitoring in evaluating of the response of a cardiovascular system of healthy volunteers was performed. The research was oriented to changes of a space weather parameters in aurural (Tixie), subauroral (Yakutsk) and medium (Saratov) areas. In each of the experimental groups there was revealed an effect of synchronization between repolarization processes of ventrical myocard responding (according to a $\mathrm{T}$-wave symmetry coefficient of a cardiogram) and geomagnetic activity (according $\mathrm{Kp}$-index). At rest the group effect of synchronization (GES) of myocard in geomagnetic activity change was noticed in $33,3 \%-61,3 \%$ of the respondents. The origin of GES has features depending on the area of habitation and an age of the volunteers.

The study is performed with the partial financial support in partnership with Russian-Ukrainian grant RFFI №14-02-90424 ukr_a.
\end{abstract}

Keywords: geomagnetic activity, cardiovascular system, T-wave symmetry coefficient.

\section{Introduction}

Changes in the pulse of a geomagnetic field of the Earth caused by active processes on the Sun and in the magnetosphere of the Earth [1] are one of the most significant factors of an adaptation failure and acute exacerbation of chronic diseases. It's known that a healthy person can stand it without pain. Revealing of the peculiarities of an adaptation of a healthy cardiovascular system to geomagnetic activity changes will allow to elaborate a number of preventing cardiologic measures.

Aim: to reveal a group response of a cardiovascular system in geomagnetic activity change at a long monitoring of healthy volunteers from different areas of habitation. The study was performed as an international multilatitudinal synchronous biophysical experiment "Heliomed".

\section{Results and discussing}

Monitoring of a cardiovascular system state was organized simultaneously in aurural (Tiksi - 11 people), subauroral (Yakutsk - 2 groups with 18 and 15 people) and medium (Saratov -31 people) areas. An average age of the volunteers was in different groups: $51,3 \pm 3,8$ years (Tixie), 43,0 $\pm 2,5$ years (Yakutsk-1), 21,6 $\pm 1,7$ years (Yakutsk-2) and 40,6 $\pm 2,2$ years (Saratov).

The duration of screening was 60 days in spring period March - April in 2014. the daily control of the repolarization processes of ventrical myocard according of ECG with a T-wave symmetry coefficient evaluation (TSC), with the help of Phazagraph device (Ukraine). The given method is good at screening of an ischemic heart disease and evaluation of changes of a space weather parameters, especially, of a solar activity, in morphological parameters of a cardiac muscle [2-4]. The daily research protocol included 4 measures of TSC: initial (at rest), after emotional load, after a physical load, at restoration after a 10-minute rest, blood pressure measuring.

Geomagnetic activity was estimated by the global geomagnetic perturbations - Kp-index, which had been detected every day during all the period of studying.

To evaluate the dynamics of the myocard state of the volunteers in geomagnetic perturbations change we had introduced the parameter "group effect of synchronization - "GES" [5], which reflected the coincidence between TSC and Kp-index maximums. If there is a presence of TSC-Kp synchronization of $66,7 \%$ from a total number of $\mathrm{Kp}$ maximums, it is registered into a GES group. It's proved that GES is different at rest and at various types of load [5]. In the present study the results of GES of volunteers from different areas were analyzed: examinees were at rest initially and at rest after a 10-min rest after a physical load. 


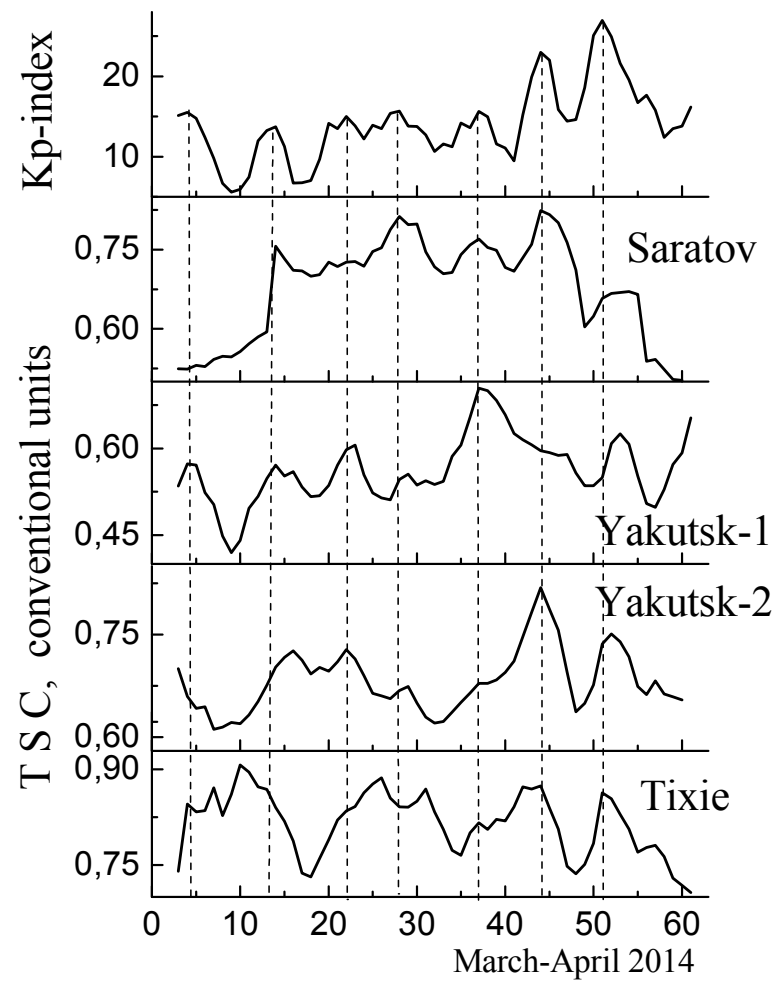

Figure: Kp-index and TSC on the different latitudes

It was noticed that GES was in all groups of volunteers. In the medium areas it was $61,3 \%$ of volunteers (Saratov), in subauroral - 33,3\% (Yakutsk-1) and 39,0\% (Yakutsk2 ), in auroral $-45,5 \%$ (Tixie). There was no statistically significant difference between these parameters $(p>0,05)$, which may be connected with a low number of samplings. So, the presented analysis isn't able to confirm that various regions are different in the proportion of "sensible" and "insensible" volunteers.

Together with this, it was discovered that GES has peculiarities connected with the regions of screening and the age of the examinees (Figure). During the period of studying there had been noticed $7 \mathrm{Kp}$ maximums, the highest levels of the geomagnetic perturbations had been fixed at the $6^{\text {th }}$ and the $7^{\text {th }}$ maximums (Figure). In the medium areas (Saratov) the TSC-Kp synchronization was at $2,4,5,6^{\text {th }} \mathrm{Kp}$ maximums and was absent in the strongest - the $7^{\text {th }}-$ maximum. Probably, the response on the strong enough $6^{\text {th }}$ maximum led to the reducing of the adaptation ability of a cardiovascular system, which became evident in the absence of a TSC and $\mathrm{Kp}$ synchronization in the $7^{\text {th }} \mathrm{Kp}$ maximum.

In subauroral areas (Yakutsk-1, Yakutsk-2) GES is different from the medium areas, as well as between the groups of various age. The volunteers of an average age (Yakutsk-1) demonstrated GES at the $1,2,3,4,5^{\text {th }} \mathrm{Kp}$ maximums. There was no response at the $6^{\text {th }} \mathrm{Kp}$ maximum, but there was - at the stronger $7^{\text {th }} \mathrm{Kp}$ maximum, although, it was not at the day of the geomagnetic perturbations. It was 2 days later. In the group of the young volunteers (Yakutsk-2) the TSC-Kp synchronization had been registered at the $6^{\text {th }}$ and the $7^{\text {th }}$ $\mathrm{Kp}$ maximums, and it was also 1 day later in the $7^{\text {th }}$ maximum. In the given group there was also noticed the $\mathrm{TSC}$ and $\mathrm{Kp}$ synchronization in the $3^{\text {rd }} \mathrm{Kp}$ maximum.

Interesting regularity was revealed in GES in auroral areas (Tixie). TSC and Kp synchronization was noticed only at the strongest $\mathrm{Kp}$ maximums - the $6^{\text {th }}$ and the $7^{\text {th }}$ (Figure). There was no myocard response on the other, not as strong geomagnetic perturbations.

The analyzing of the TSC-Kp synchronization effect in different groups of studying shows that the leading factor for synchronization of a myocard state and geomagnetic perturbations is a value of a $\mathrm{Kp}$-index, as well as a state of adaptation abilities of a cardiovascular system of the volunteers.

\section{Conclusions}

The group effect of the TSC-Kp synchronization is revealed at all studying areas (medium, subauroral and auroral). The degree of incidence of volunteers with myocard "sensitive" to geomagnetic perturbations doesn't depend on the region of habitation. At the same time, the origin of GES has peculiarities in various - medium, subauroral and auroral - areas. The groups of volunteers of different age, living in the same area, are also characterized by different variants of myocard response on geomagnetic activity change. Besides, an initial state of adaptation ability of a cardiovascular system of the volunteers also determines peculiarities of myocard state response on geomagnetic activity change.

The study performed with the financial support of collaboraive Russian-Ukrainian grant 14-02-90424 «The role of normal and extreme heliogeophysical processes in evolution of biosphere».

\section{References}

1. Obridko V.N. et al.: 2014, Psychosomatic and integrative research, 1, http://pssr.pro/articles/237.

2. Samsonov S.N.: 2013, Odessa Astron. Publ., 26, 297.

3. Fainzilberg L.S.: 1998, Control systems and machines, 4, 40 .

4. Vishnevsky V.V. et al.: 2003, Biomedical technologies and radioelectronics, $\mathbf{3}, 3$.

5. Parshina S.S. et al.: 2015, IT + M\&Ec'2015, Spring session, 316, http://glorioz.com/doki/me/2015/2015_5.pdf. 